\title{
$\mathbf{T}$ 型接头角接静轴肩摚拌摩擦焊缺陷研究*
}

\author{
曾申波 ${ }^{1,2}$ 陈高强 ${ }^{1,2}$ 刘矍 1,2 张 弓 1,2 史清宇 ${ }^{1,2}$ \\ (1. 清华大学机械工程系 北京 100084 ;
}

2. 清华大学先进成形制造教育部重点实验室 北京 100084)

\begin{abstract}
摘要: 通过一系列工艺参数试验, 获得无缺陷的 $\mathrm{T}$ 型接头焊缝。针对缺陷的形成, 重点分析三种不同的摚拌针, 即圆锥光针、 圆雉螺纹针和短圆柱光针, 对 $6 \mathrm{~mm}$ 厚的 6082-T6 铝合金 $\mathrm{T}$ 型接头从内角角焊缝进行静轴肩摚拌摩擦焊接, 并分析对应的焊 缝产生的宏观缺陷。发现角接静轴肩傥拌摩擦焊容易在前进侧产生孔洞或沟槽缺陷。进一步分析发现, 缺陷的产生受 $\mathrm{T}$ 接摚 拌针和 $\mathrm{T}$ 接静轴肩的影响, 在搅拌头周围, 受 $\mathrm{T}$ 接静轴肩和 $\mathrm{T}$ 接摚拌针上部的影响, 会形成摚拌针上部塑性流动区域, 受摚 拌针下部的影响, 会形成摚拌针下部塑性流动区域. 通过研究提出: 摚拌针下部的孔洞缺陷可以通过采用螺纹的锥形摚拌针 进行消除。而摚拌针上部的孔洞缺陷受到 $90^{\circ}$ 的 $\mathrm{T}$ 接静轴肩几何形状的影响流动能力较弱, 通过倾角的改变可获得无缺陷的 焊缝, 并且对其缺陷形成机制的物理模型进行分析。
\end{abstract}

关键词: $\mathrm{T}$ 型接头; 角接静轴肩摚拌摩擦焊; 倾角; 缺陷; 形成机制

中图分类号: TG453

\section{Study on Defects of T-joints by Corner Stationary Shoulder Friction Stir Welding}

\author{
ZENG Shenbo $^{1,2}$ CHEN Gaoqiang ${ }^{1,2}$ LIU Qu ${ }^{1,2}$ ZHANG Gong ${ }^{1,2}$ SHI Qingyu ${ }^{1,2}$
}

(1. Department of Mechanical Engineering, Tsinghua University, Beijing 100084;

2. Key Laboratory of Advanced Material Processing Technology, Ministry of Education of China,

Tsinghua University, Beijing 100084)

\begin{abstract}
The defect free T-joints obtained by corner stationary shoulder friction stir welding. Three different pins, i.e. tapered pin, tapered pin with thread, short cylindrical pin, are used for friction stir welding of $6 \mathrm{~mm}$ thick 6082-T6 T-joint aluminum alloy from the inner corner weld, and corresponding analysis of the macro defects of the weld is carried out. It is found that the tunnel or groove defects are easily produced on the advancing side of the corner joint during friction stir welding. The defects are affected by the pin and corner stationary shoulder. The plastic material flow region on the upper part of pin will be formed around tool under the influence of the corner stationary shoulder and the upper part of the pin. The plastic material flow region on the lower part of the pin will be formed under the influence of the lower part of the pin. Besides, the tunnel defects on the upper part of the pin is affected by the stationary shoulder of $90^{\circ}$ profile corner stationary shoulder, The defect free T-joints can be obtained by changing the tool tilt angle, and the physical model of the defect formation mechanism is analyzed.
\end{abstract}

Key words: T-joint; corner stationary shoulder friction stir welding; material flow; defects; formation mechanism

\section{0 前言}

$\mathrm{T}$ 型接头是工程中一种常用的接头, 特别是在 航空制造业中，飞机机身等由高强铝合金组成 ${ }^{[1]}$, 可考虑焊接的方法主要是铆接、激光焊接、搅拌摩 擦焊焊接。其传统的连接工艺为铆接。该工艺不仅 增加了重量, 而且需要密封剂, 容易腐蚀。而采用 激光焊接的方法, 从内角进行焊接, 容易产生气孔、

* 国家自然科学基金资助项目(51705280,51375259)。20190528 收到初稿, 20190929 收到修改稿
裂纹等缺陷 ${ }^{[2]}$ 。此外, 采用传统的搅拌摩擦焊方法, 从外侧进行焊接也是近年来的研究热点 ${ }^{[3-7]}$, 但焊接 过程中容易产生孔洞和弱结合。并且, 外侧底板容 易减薄, 工装的圆角处容易产生缺陷。因此, 需要 开发一种新型的焊接工艺方法, BUFFA 等 ${ }^{[8-10]}$ 提出 了从内角角焊缝焊接 $\mathrm{T}$ 型接头的工艺, 但目前还停 留在工艺探索阶段, 对焊缝的成形机理, 缺陷研究 都还比较少 ${ }^{[11]}$, 需要进一步研究。

现有的研究表明, 采用内角静轴肩搅拌摩擦焊 焊接 $\mathrm{T}$ 型接头, 由于其轴肩静止, 具有很少减薄的 光滑焊缝表面、焊缝界面一致和几乎线性的热输入 
等优点。因此, 开展内角进行焊接的 $\mathrm{T}$ 型接头具有 重要的意义。国内有部分科研院所进行了 $\mathrm{T}$ 型接头 的探索和研究, 吉华等 ${ }^{[12]}$ 开展了 $\mathrm{T}$ 型接头的组织及 力学行为研究。李丽茹等 ${ }^{[13]}$ 针对船用铝合金角接的 固定轴肩摚拌摩擦焊焊具进行了设计研究, 并进行 了 $\mathrm{T}$ 型接头的试验研究, 获得了无缺陷的 $\mathrm{T}$ 型接头。 国内外, 在工程上非常重视静轴肩摚拌摩擦焊的开 发和利用。目前初步实现了角接静轴肩搅拌摩擦焊 的焊接 ${ }^{[14]}$, 但关于搅拌针几何形状对接头缺陷的形 成机制以及获得无缺陷焊缝的原因基本没有报道。 特别地, 对于不同搅拌针几何形状, 对缺陷的影响 和形成机制也没有做进一步的深入研究和对比。

因此, 本文首先通过一系列的工艺试验获得无 缺陷的 $\mathrm{T}$ 型接头焊缝。焊后进行分析, 针对缺陷的 形成, 采用不同形状的摚拌针进行 $\mathrm{T}$ 型接头的摚拌 摩擦焊接, 详细探讨不同形状傥拌针的接头焊缝成 形和缺陷情况, 在此基础上, 进一步对缺陷进行分 析, 阐明其产生的机理, 提出相关的物理模型。本 文工作将有利于 $\mathrm{T}$ 型接头角接静轴肩摚拌摩擦焊的 科学研究和工程应用。

\section{1 试验材料及方法}

试验采用自制的搅拌焊具和工装, 在 XTM-900 立式数控摚拌摩擦焊机进行。待焊底板尺寸为 200 $\mathrm{mm} \times 155 \mathrm{~mm} \times 6 \mathrm{~mm}$, 筋板尺寸为 $200 \mathrm{~mm} \times 73 \mathrm{~mm} \times$ $6 \mathrm{~mm}$, 其材料为 6082-T6 铝合金, $\mathrm{T}$ 型接头的摚拌 摩擦焊接示意图, 如图 1 所示。

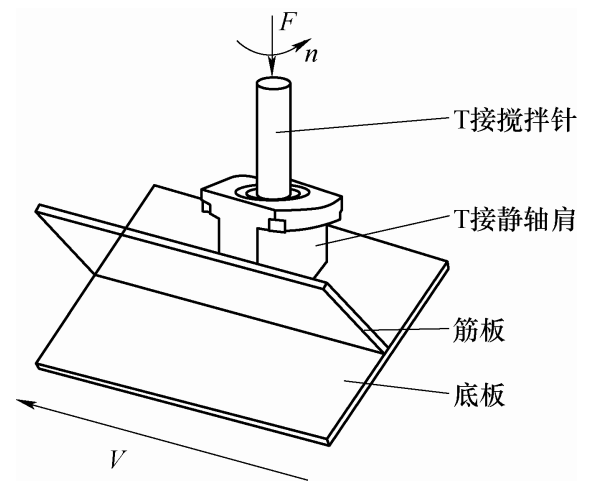

图 $1 \mathrm{~T}$ 型接头焊接示意图

试验过程中, 为了获得无缺陷的焊缝, 在倾角 为 $0^{\circ}$ 时, 设计了各种不同的摚拌针进行焊接, 相应 的采用了不同的焊接转速和焊接速度, 如图 $2 \mathrm{a}$ 所 示, 所用的摚拌针如下: 光针(圆雉光针)、雉针 (圆 雉螺纹针)、正反螺纹针、圆柱针、圆环针、四棱针, 其主要区别是搅拌针不同的结构形状。在倾角为 $1^{\circ}$ 时, 如图 $2 b$ 所示, 采用圆雉形的摚拌针获得了无缺 陷的焊缝, 同时发现, $\mathrm{T}$ 型接头孔洞缺陷易于在前
进侧上部形成缺陷。从图 2 中可以才看出, 在倾角 为 $0^{\circ}$ 时, 采用不同的摚拌针进行焊接, 焊缝成形存 在不同的表面宏观缺陷, 主要是前进侧出现沟槽缺 陷, 而在倾角为 $1^{\circ}$ 时, 采用圆雉螺纹针, 在一定的 工艺参数范围内, 可获得无缺陷的焊缝。进一步分 析发现, 摚拌针几何形状影响焊缝的缺陷成形。

表面宏观缺陷



(a) 倾角为 $0^{\circ}$ 时的工艺参数

表面宏观缺陷

$\triangle$ 前进侧内部孔洞



(b) 倾角为 $1^{\circ}$ 时的工艺参数

图 2 焊接工艺参数及接头缺陷

为了对缺陷的形成机制进行深入分析，并获得 无缺陷的焊缝, 需要通过对搅拌针形状的改进设计, 研究其焊缝成形规律。试验中重点分析三种不同形 状的搅拌针进行焊接, 分别是圆锥光针、圆雉螺纹 针、短圆柱光针，示意图如图 3 所示(图中， $\mathrm{T}$ 接摚 拌针与 $\mathrm{T}$ 接静轴肩间隙 $0.3 \mathrm{~mm}$, 搅拌针上部直径为 $\phi 8 \mathrm{~mm}$, 搅拌针下部顶部直径为 $\phi 4 \mathrm{~mm})$ 。试板固 定在工装上, $\mathrm{T}$ 接摚拌头从试板一侧焊接完后, 调 转试板, 焊接另一侧, 实现 $\mathrm{T}$ 型接头的两道次焊缝 的焊接。获得的试样分别为两道次焊接的圆锥光针 $\mathrm{T}$ 型接头试样、两道次焊接的圆雉螺纹针的 $\mathrm{T}$ 型接
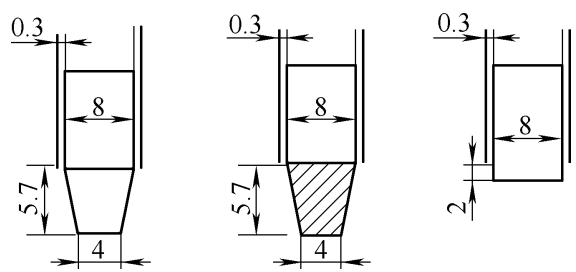

图 3 不同形状的摚拌针 
头试样, 以及第一道次焊接圆雉螺纹针、第二次道 次焊接短圆柱光针的 $\mathrm{T}$ 型接头对比试样, 焊接工艺 参数均如下: 主轴转速 $1500 \mathrm{r} / \mathrm{min}$, 焊速 100 $\mathrm{mm} / \mathrm{min}$, 无倾角焊接, 下压量为 $0.4 \mathrm{~mm}$ 。

焊后, 采用 NH7740B 线切割设备从试样横截 面进行取样, 接头试样磨平抛光后采用 Keller 试剂 进行腐蚀, 之后用 MEFEA 体式显微镜和光学显微 镜 OLYMPUS-BX51M 进行观察。

\section{2 试验结果与讨论}

\section{$2.1 \mathrm{~T}$ 型接头焊缝成形及横截面形貌观察}

图 $4 \mathrm{a}$ 为在倾角为 $0^{\circ}$ 时采用圆雉光针焊接的焊 缝表面, 从图中可以看出, 角接焊缝表面成形良好, 其表面没有明显的缺陷, 铝合金材料在 $90^{\circ} \mathrm{T}$ 接静 轴肩后沿的顶锻作用下滑过焊缝表面。同时, 在摚 拌针逆时针旋转作用下, 摩擦生热软化的材料在前 进过程中发生了塑性流动并填充煌缝。焊后, 摚拌 头退出, 匙孔上端保留着几乎与搅拌针上部直径尺 寸相当的材料残余。

在倾角为 1 时, 获得了无缺陷的 $\mathrm{T}$ 型接头, 其 外观形貌如图 4b 所示, 图 4c 为其焊缝横截面图, 焊缝无缺陷, 总体来看, 表面光滑, 基本没有减薄, 同时, 焊缝横截面前进侧后退侧较为均匀对称。

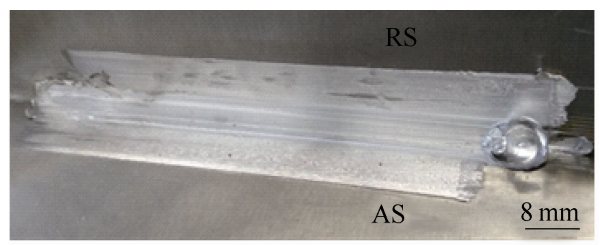

(a) 圆锥光针且倾角为 $0^{\circ}$

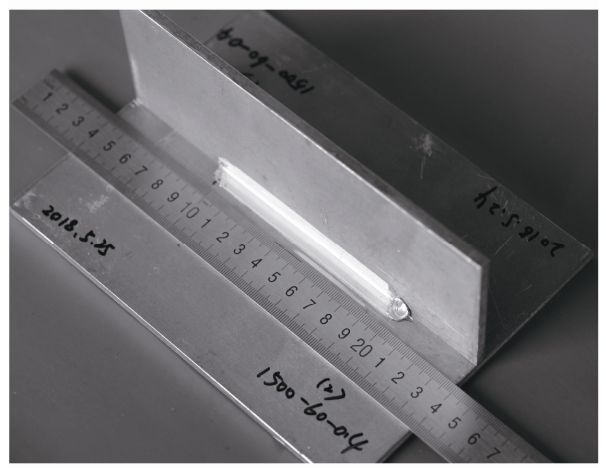

(b) 圆雉螺纹针且倾角为 $1^{\circ}$

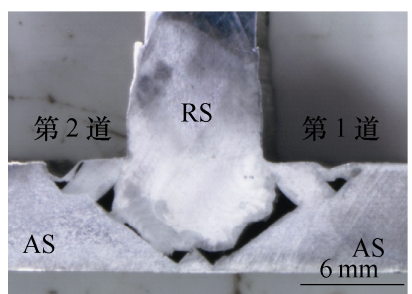

(a) 圆雉光针

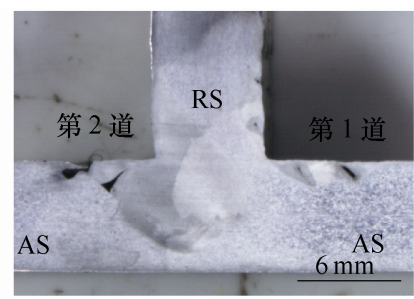

(b) 圆雉螺纹针

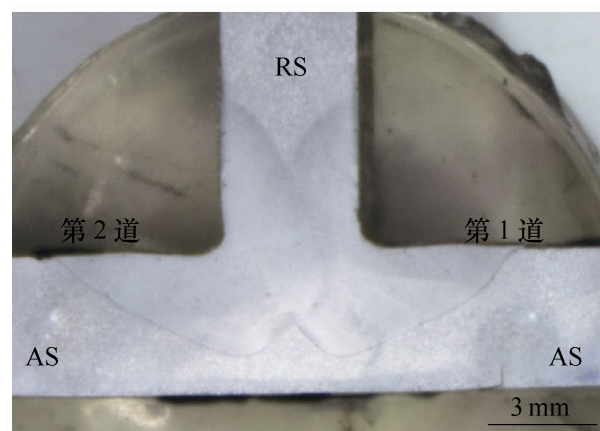

(c) 为图 $4 \mathrm{~b}$ 的焊缝横截面

图 4 焊缝外观形貌
图 5 为三种不同摚拌针的通过两道次焊接所获 得的 $\mathrm{T}$ 型接头焊缝横截面图, 观察每道次焊缝横截 面, 发现, 每道焊缝前进侧的界面清晰, 后退侧界 面的模糊, 这与柯黎明等 ${ }^{[15-16]}$ 进行传统的摚拌摩擦 焊的结果几乎一致。但相比传统的动轴肩搅拌摩擦 焊, 静轴肩摚拌摩擦焊 $\mathrm{T}$ 型接头的单道次焊缝前进侧 和后退侧几乎一致的线性热输入, 焊缝横截面几乎没有 减薄, 摚拌针上部受 $\mathrm{T}$ 接静轴肩影响下的大区域流 场没有出现 ${ }^{[17]}$ 。

进一步对比不同搅拌针焊后试样的横截面形貌。 发现所有试样通过两次顺序焊接后, 都实现了 $\mathrm{T}$ 型接 头的焊接, 但从图 5a 中可以看出, 圆雉光针焊后的焊 缝底板上出现了沿筋板对称, 且几乎一致的孔洞缺陷。 该缺陷的位置都位于两道次焊接焊缝的前进侧(AS), 而在后退侧(RS)焊缝未发现明显缺陷。如图 $5 b$ 所示, 圆锥螺纹针焊接的焊缝也出现了沿筋板对称, 且几乎 一致的细小孔洞缺陷, 且均出现在摚拌针与轴肩结合 处的上部, 也就是搅拌针中上部区域。该缺陷的位置 也位于两道次焊缝的前进侧。与之不同的是, 圆雉螺 纹针下部没有明显的缺陷。进一步对图 5c 分析, 该图 第一道焊缝采用同样的螺纹圆锥针焊接, 也产生的如 图 $5 \mathrm{~b}$ 所示的孔洞缺陷, 但在焊接第二道焊缝时, 采 用较短的圆柱摚拌针焊接, 该焊接位置位于 $\mathrm{T}$ 接搅拌 针和 $\mathrm{T}$ 接静轴肩相结合部分, 也就是与底板和筋板高 度贴合部位, 焊后发现产生了同样的孔洞焊接缺陷, 而且位于前进侧。这也表明, 在工艺参数保持不变的 基础上, 发现圆雉螺纹针能很好地消除摚拌针下部前 进侧的孔洞缺陷, 但就算采用短圆柱光针进行二次 焊接, 摚拌针上部前进侧的孔洞缺陷仍然难以消除。

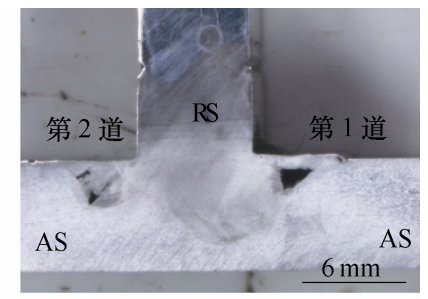

(c) 短圆柱光针第二道焊缝

图 5 焊缝横截面形貌 


\section{$2.2 \mathrm{~T}$ 型接头焊接缺陷的形成}

为了便于观察和更好地分析摚拌针对缺陷形成 的影响, 对图 5 的不同搅拌针焊后第二道焊缝进行 试样顺时针旋转 $45^{\circ}$ (上图为旋转前位置, 下图为对 应的旋转后的位置), 如图 6 所示。对比图 $6 \mathrm{a}$ 和图 $6 \mathrm{~b}$ 可以看出, 圆雉光针焊接时, 焊缝前进侧上部和 下部(也就是摚拌针前进侧的上部和下部)沿搅拌针 前进侧边沿出现了几乎连续的孔洞缺陷(如图中 $\mathrm{X}$ 形位置所示), 而采用圆雉螺纹针后, 如图 $6 \mathrm{~b}$ 所示,
则仅在焊缝前进侧的中上部出现了细小孔洞(也就 是摚拌针前进侧上部)。这说明在搅拌针下部增加螺 纹后, 增加了塑性材料的驱动力, 改变了材料的流 动形式, 促进了材料的流动 ${ }^{[18-19]}$, 这一现象也能在 文献[18-19]中得到印证，从而消除了搅拌针下部前 进侧的孔洞缺陷。为了进一步研究搅拌针上部的焊 接缺陷, 减少搅拌针下部对搅拌针上部的影响, 采 用较短的圆柱摚拌针进行焊接, 焊缝位置为搅拌针 上部与 $\mathrm{T}$ 接静轴肩旋转结合的部位。

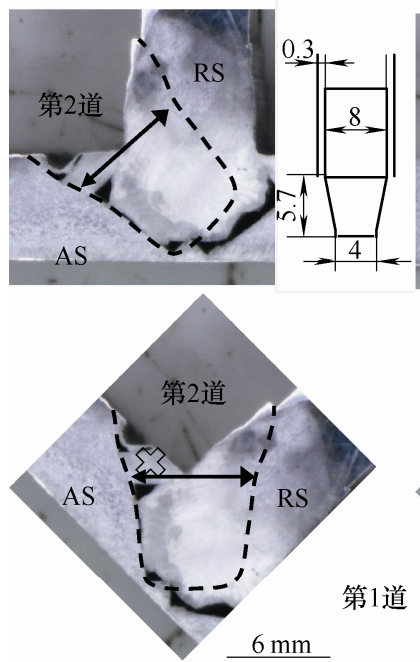

(a) 圆雉光针

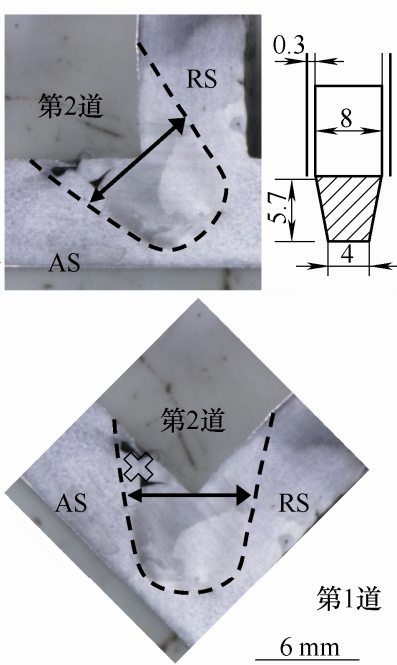

(b) 圆锥螺纹针

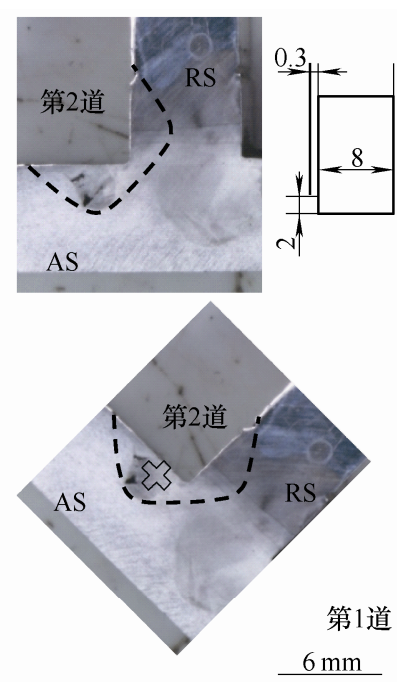

(c) 短圆柱光针

图 6 不同针的接头横截面形貌对比

图 6c 所示, 通过焊接, 从图中可发现, 搅拌针 上部依然出现了类似的孔洞缺陷。也表明在其他条 件不变的情况下, 搅拌针上部的焊接缺陷与搅拌针 下部的焊接缺陷形成机制可能有所不同。缺陷的形 成与搅拌针上部的形状关系不紧密, 从焊具的结构和 形状上观察, 上部缺陷的形成与角接静轴肩搅拌摩擦 焊的独特结构有关。一是 $90^{\circ}$ 的 $\mathrm{T}$ 接静轴肩在焊缝形 成过程中通过 $90^{\circ}$ 的静轴肩后沿对焊缝进行顶锻焊 合。其次, 要保持摚拌针旋转和 $\mathrm{T}$ 接静轴肩静止, 摚 拌针与 $\mathrm{T}$ 接静轴肩穿接处存在相贯区, 再一个搅拌针 与 $\mathrm{T}$ 接静轴肩穿接配合处存在配合间隙 $0.3 \mathrm{~mm}$, 如图 2 所示, 从而影响了搅拌针上部的塑性材料的流动。 因此, $\mathrm{T}$ 型接头在搅拌针上部更加容易产生缺陷, 这 也为 $\mathrm{T}$ 接搅拌针和 $\mathrm{T}$ 接静轴肩的设计提供了新的难 题。因此, 要实现 $\mathrm{T}$ 型接头的无缺陷焊接, 必须要了 解接头缺陷的形成机制, 特别是 $\mathrm{T}$ 接静轴肩和 $\mathrm{T}$ 接摚 拌针相贯区的结合部位的缺陷形成机理。需要对摚拌 针中上部区域材料流动机理与缺陷形成进行机制分 析, 深入阐明 $\mathrm{T}$ 接静轴肩和 $\mathrm{T}$ 接搅拌针结构形状与热 力耦合作用下的塑性流动的材料作用机制。

进一步研究发现, 图 7 为焊后, $\mathrm{T}$ 接搅拌焊具 的搅拌针和 $\mathrm{T}$ 接静轴相贯区部位实物图。从图中可 以看出, 在轴肩和搅拌针的结合部(虚线表示), $\mathrm{T}$
接摚拌针高速旋转, $\mathrm{T}$ 接静轴肩静止, 在 $\mathrm{T}$ 接静轴 肩前沿和后沿都有塑性材料流动的痕迹, 过渡区域 也经历了摩擦和磨损。这表明, $\mathrm{T}$ 接静轴肩和 $\mathrm{T}$ 接 搅拌针在摚拌摩擦过程中经历了很大的温度变化和 复杂的热力耦合环境, 搅拌摩擦焊的下压力通过 $\mathrm{T}$ 接静轴肩施加于焊缝表面, 摚拌针对焊缝材料摩擦 生热, 并驱动材料产生塑性流动。

\section{$2.3 \mathrm{~T}$ 型接头缺陷形成的物理模型}

以上, 通过改变不同的摚拌针形状进行焊接, 相应对比分析焊缝横截面形貌, 发现, 在 $\mathrm{T}$ 型接头 角接静轴肩搅拌摩擦焊焊接过程中, 塑性材料流动 过程由搅拌针中上部流动区和搅拌针下部流动区组 成。对于搅拌针中上部流动区，材料直接受静止的 $\mathrm{T}$ 接静轴肩和旋转的 $\mathrm{T}$ 接摚拌针影响而发生塑性变 形。根据金属塑性加工宏观规律中的阻力最小定律, 即物体的各质点会沿阻力最小的方向流动, $\mathrm{T}$ 型接 头焊接过程中, $\mathrm{T}$ 接搅拌针旋转摩擦, 附近材料受 热围绕摚拌针流动, 并最终流向 $\mathrm{T}$ 接静轴肩后沿, 在封闭区域中形成焊缝, 与塑性加工的工况环境类 似。由此可知后方存在一个压力最小的区域。孔洞 的缺陷发生在前进侧, 也表明前进侧的压力最低。 孔洞缺陷即是塑性材料未完全填充的空腔, 即搅拌 针摩擦剪切周围材料受到类似切削形成的空腔, 其 
形成机制可描述为类似切削形成空腔。

在一定的焊接转速和焊接速度下, $\mathrm{T}$ 接搅拌针 与被焊材料摩擦产热, 产生了一定的热输入, 使 $\mathrm{T}$ 接搅拌头周围材料发生软化。同时, $\mathrm{T}$ 接搅拌头周 围材料受到焊缝表面结合的压力作用。通过观察前 进侧中上部的焊缝横截面形貌, 可知前进侧中上部 材料的孔洞缺陷主要是由于 $\mathrm{T}$ 接搅拌针上部的材料 流动不足产生的。如图 8 所示。图 $8 \mathrm{a}$ 中, 前进侧孔 洞缺陷包括 $\mathrm{T}$ 接静轴肩附近的上部孔洞缺陷和中部 孔洞缺陷, 其中中部孔洞缺陷是由于来自搅拌上部 流动的材料和来自搅拌针下部的材料汇流后, 不能 很好的结合而形成的孔洞, 见图 $8 b$ (图 $8 b$ 为图 $8 \mathrm{a}$



图 $7 \mathrm{~T}$ 接搅拌针上部与 $\mathrm{T}$ 接静轴肩配合

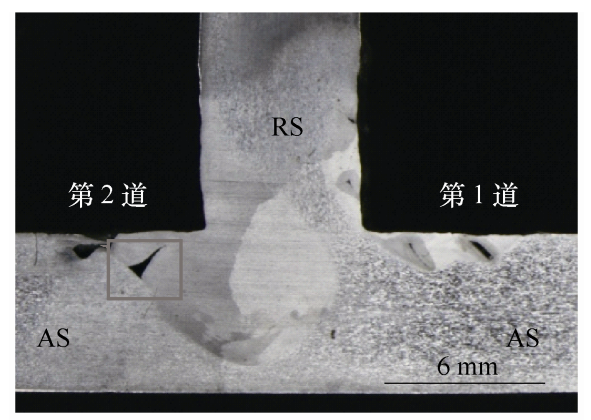

(a) 圆锥螺纹针

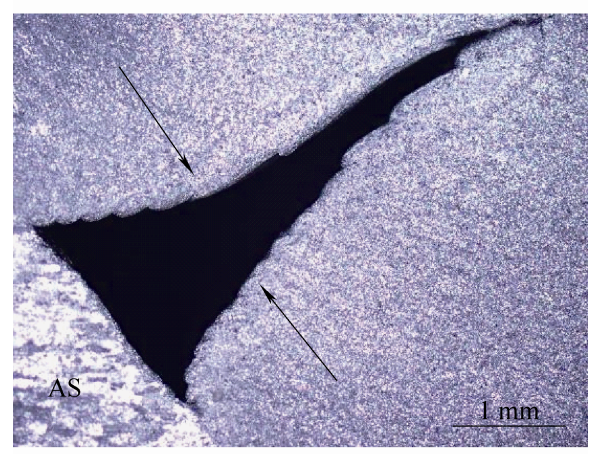

(b) 前进侧中部孔洞缺陷的形成

图 $8 \mathrm{~T}$ 型接头前进侧缺陷附近材料流动



图 $10 \mathrm{~T}$ 型接头前进侧中上部孔洞缺陷形成物理模型
的实线方框的局部放大图), 该缺陷本质上也是由焊 缝前进侧材料的流动不足造成的, 该过程可描述为: 塑性材料填充, 流入焊缝前进侧。

图 9 所示为 $\mathrm{T}$ 型接头前进侧的受力和缺陷形成 分析(图中实线为 $\mathrm{T}$ 接静轴肩示意图, 虚线为搅拌针 的示意图)。 $\mathrm{T}$ 型接头前进侧缺陷形成时, 轴肩会对 焊缝表面施加的顶锻力。从图中可以看出, 焊缝表 面受到来自于 $\mathrm{T}$ 接静轴肩 $90^{\circ}$ 侧面的顶锻力, 该顶 锻力是主轴顶锻力的一个 $45^{\circ}$ 方向上的侧向力(前进 侧受力 $F_{\mathrm{AS}}$ 、后退侧受力 $F_{\mathrm{RS}}$ ), 该侧向力分别与前 进侧表面和后退侧表面垂直，这与传统的主轴顶锻 力垂直于焊缝表面不同。在侧向力的作用下， $\mathrm{T}$ 接 静轴肩对其两侧焊缝完成顶锻焊合, 当 $\mathrm{T}$ 接静轴肩 与 $\mathrm{T}$ 接试样装配有偏差时, 将会导致两侧受力不均 匀, 因此, 在焊接时, 要确保 $\mathrm{T}$ 接静轴肩与其接触 的焊缝表面高度贴合，才能实现焊缝的顶锻焊合。

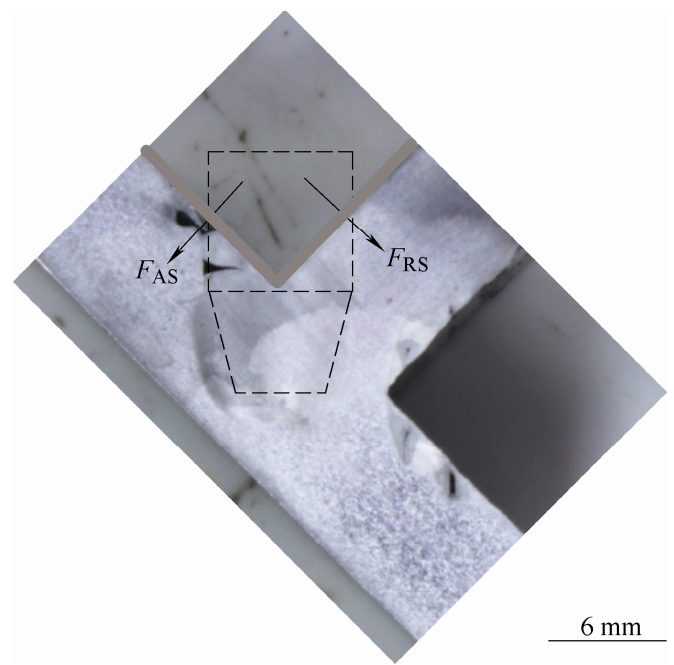

图 $9 \mathrm{~T}$ 型接头受力分析

基于以上观察和分析, 本文采用图 10 中的模型 对 $\mathrm{T}$ 型接头前进侧中上部缺陷形成过程进行描述, 其缺陷形成机制可描述如下。

首先, $\mathrm{T}$ 接摚拌针和母材在前进侧摩擦、剪切, 塑 性材料经过 $\mathrm{T}$ 接静轴肩前沿, 大部分材料随搅拌针旋转 流向后退侧，形成空腔。另一方面，后退侧材料在经过 $\mathrm{T}$ 接静轴肩后沿，流向前进侧空腔。当材料热输入不足 时, 前进侧会形成贯通的沟槽。当材料流入不足时, 在 $\mathrm{T}$ 接静轴肩压力的作用下，焊缝表面形成沟槽，内部形 成孔洞。当材料流入足够时, 焊缝成形完好。
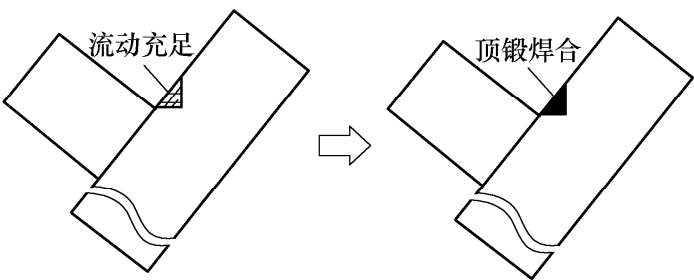


\section{3 结论}

(1) $\mathrm{T}$ 型接头角接静轴肩搅拌摩擦焊时, 可获得 表面成形良好、无明显缺陷焊缝。但在焊缝区内部 搅拌针中上部易形成孔洞缺陷, 其位置在焊缝的前 进侧。

(2) $\mathrm{T}$ 接静轴肩轴肩基本不产热, 只在表面摩擦 形成光滑焊缝, 且角度为 $90^{\circ}, \mathrm{T}$ 接搅拌针旋转提供 驱动力和金属迁移, 试验发现易于在焊缝的前进侧 中上部产生孔洞缺陷, 焊缝区前进侧由搅拌针下部 的塑性材料和摚拌针上部的塑性材料汇流而成。

(3) 不同的摚拌针对周围金属的驱动力和流动 有重要影响。对比发现, 圆雉螺纹针可显著增强材 料流动能力, 并形成摚拌针下部无缺陷的焊缝。圆 锥光针下部流动能力较弱, 易于在整个前进侧形成 孔洞。

（4）通过简易的前进侧中上部孔洞缺陷形成机 制物理模型, 描述了 $\mathrm{T}$ 型接头角接静轴肩摚拌摩擦 焊的焊缝金属塑性流动方式和缺陷的形成过程。

(5) 对于 $\mathrm{T}$ 型接头角接静轴肩摚拌摩擦焊, 摚 拌针下部的圆雉螺纹针相比圆雉光针更有利于无缺 陷焊缝的形成, 同时发现倾角对获得无缺陷的焊缝 具有重要影响, 文中在倾角 $1^{\circ}$ 时, 在一定的参数范 围内可获得无缺陷的焊缝。由于倾角改变对焊缝的 形成影响因素涉及热力耦合作用的复杂过程以及流 动能力, 需要进一步更深入的分析。

\section{参 考 文 献}

[1] SHTRIKMAN M M, KORNEVICH A P, PINSKIY A V, et al. Friction stir welding of ribbed panels of aircraft airframes[J]. Welding International, 2018, 32(3): 219-222.

[2] KASHAEV N, VENTZKE V, CAM G. Prospects of laser beam welding and friction stir welding processes for aluminum airframe structural applications[J]. Journal of Manufacturing Processes, 2018, 36: 571-600.

[3] FRATINI L, BUFFA G, FILICE L, et al. Friction stir welding of AA6082-T6 T-joins: Process engineering and performance measurement, Proceedings of the Institution of Mechanical Engineers[J]. Part B : Journal of Engineering Manufacture, 2006, 220(5): 669-676.

[4] FRATINI L, BUFFA G, MICARI F, et al. On the material flow in FSW of T-joints: Influence of geometrical and tecnological parameters[J]. The International Journal of Advanced Manufacturing Technology, 2008, 44(5-6): 570-578.

[5] CUI L, YANG X, ZHOU G, et al. Characteristics of defects and tensile behaviors on friction stir welded AA6061-T4 T-joints[J]. Materials Science and Engineering: A, 2012, 543: 58-68.

[6] ZHOU G, YANG X Q, CUI L, et al. Study on the microstructures and tensile behaviors of friction stir welded T-joints for AA6061-T4 alloys[J]. Journal of Materials Engineering and Performance, 2012, 21(10): 2131-2139.

[7] CUI L, YANG X, XIE Y, et al. Process parameter influence on defects and tensile properties of friction stir welded T-joints on AA6061-T4 sheets[J]. Materials \& Design, 2013, 51: 161-174.

[8] BUFFA G, FRATINI L, ARREGI B, et al. A new friction stir welding based technique for corner fillet joints: experimental and numerical study[J]. International Journal of Material Forming ，2010，3(S1): 1039-1042.

[9] 刘会杰, 李金全, 段卫军. 静止轴肩搅拌摩擦焊的研究 进展 $[\mathrm{J}]$. 焊接学报, 2012, 33(5): 108-112.

LIU Huijie, LI Jinquan, DUAN Weijun. Research progress of static shoulder friction stir welding[J]. Transaction of the China Welding Institution, 2012，33(5): 108-112.

[10] MARTIN J P, STANHOPE C, GASCOYNE S. Novel techniques for corner joints using friction stir welding[M]// MISHRA R, MAHONEY M W, SATO Y, et al. Friction Stir Welding and Processing VI. Wiley, 2011.

[11] 刘德佳, 丁江影, 涂文志, 等. $\mathrm{T}$ 型接头搅拌摩擦焊接 的研究进展 [J]. 材料导报(A), 2016, 30(12): 68-72.

LIU Dejia, DING Jianghao, TU Wenzhi, et al. Progress in friction stir welding of T-joints[J]. Material Report(A), 2016, 30(12): 68-72.

[12] 吉华, 徐萌, 封小松, 等. 静止轴肩搅拌摩擦焊 $\mathrm{T}$ 型接 头组织及力学行为 $[\mathrm{J}]$. 电焊机, 2013, 43(9): 37-40.

JI Hua, XU Meng, FENG Xiaosong, et al. Microstructure and mechanical behavior of T-joint in static shoulder friction stir welding [J]. Welding Machine, 2013, 43(9): $37-40$.

[13] 李丽茹, 顾长石, 甘露, 等. 船用铝合金角接的固定轴 肩搅拌摩擦焊焊具设计研究 [J]. 造船技术, 2013, 316(6): 52-55.

LI Liru, GU Changshi, GAN Lu, et al. Design and research of friction stir welding tool for stationary shoulder of marine aluminum alloy angular joints[J]. Marine Technology, 2013, 316(6): 52-55.

[14] MALTIN C A, NOLTON L J, SCOTT J L, et al. The potential adaptation of stationary shoulder friction stir welding technology to steel[J]. Materials \& Design, 2014, 64: 614-624.

[15] 毛育青，柯黎明，刘奋成，等. 铝合金厚板 FSW 焊缝成 
形及金属流动行为分析[J]. 航空学报, 2016, 38(3): 1-8. MAO Yuqing, KE Liming, LIU Fencheng, et al. Weld formation and metal flow behavior of thick aluminium alloy plates[J]. Acta Aeronautical et Astronautica sinica, 2016, 38(3): 1-8.

[16] 毛育青, 柯黎明, 刘奋成, 等. 铝合金厚板摚拌摩擦焊 焊缝疏松缺陷形成机理[J]. 航空学报, 2017, 37(11): 3546-3553.

MAO Yuqing, KE Liming, LIU Fencheng, et al. Formation mechanism of weld loose defects in friction stir welding thick plates of aluminum alloy [J]. Acta Aeronautical et Astronautica sinica, 2017, 37(11): 3546-3553.

[17] DONATUS U, THOMPSON G E, ZHOU X, et al. Flow patterns in friction stir welds of AA5083 and AA6082 alloys[J]. Materials \& Design, 2015， 83: 203-213.

[18] 王善林, 柯黎明, 潘际銮, 等. 搅拌针形状对摚拌摩擦 焊焊缝截面形貌的影响 [J]. 焊接学报, 2007, 28(5):
34-37.

WANG Shanlin, KE Liming, PAN Jiluan, et al. Effect of stirring pin shape on cross-section morphology of friction stir welding $[\mathrm{J}]$. Transaction of the China Welding Institution, 2007, 28(5): 34-37.

[19] 柯黎明, 潘际刑, 邢丽, 等. 摚拌摩擦焊焊缝金属塑性 流动的抽吸-挤压理论[J]. 机械工程学报, 2009, 45(4): 89-94.

KE Liming , PAN Jiluan, XING $\mathrm{Li}$, et al. Suction-extrusion theory of metal plastic flow in friction stir welding seam[J]. Journal of Mechanical Engineering, 2009, 45(4): 89-94.

作者简介: 曾申波, 男, 1980 年出生, 博士研究生。主要研究方向为搃 拌摩擦焊焊接。

E-mail: csb14@mails.tsinghua.edu.cn

史清宇(通信作者), 男, 1971 年出生, 博士, 长聘教授, 博士研究生导 师。主要研究方向为摚拌摩擦焊焊接与加工, 焊接力学与冶金。

E-mail: shiqy@mail.tsinghua.edu.cn 